\title{
Female Genital Grading Table
}

National Cancer Institute

\section{Source}

National Cancer Institute. Female Genital Grading Table. NCI Thesaurus. Code C157688.

A table within the standard terminology developed by the NIH Division of AIDS (DAIDS)

to report expedited adverse events (EAEs) reported by Clinical Research Sites (CRSs)

participating in DAIDS-supported studies. This table contains specific adverse event grading terminology for Female Genital Microbicide Studies. 